\title{
Resonant-wavelength control of nanocavities by nanometer-scaled adjustment of two-dimensional photonic crystal slab structures
}

\section{$\operatorname{AUTHOR}(\mathrm{S}):$}

Song, Bong-Shik; Nagashima, Takuji; Asano, Takashi; Noda, Susumu

\section{CITATION:}

Song, Bong-Shik ...[et al]. Resonant-wavelength control of nanocavities by nanometerscaled adjustment of two-dimensional photonic crystal slab structures. IEEE PHOTONICS TECHNOLOGY LETTERS 2008, 20(5-8): 532-534

\section{ISSUE DATE:}

2008-03

URL:

http://hdl.handle.net/2433/84558

\section{RIGHT:}

(c) 2008 IEEE. Personal use of this material is permitted. However, permission to reprint/republish this material for advertising or promotional purposes or for creating new collective works for resale or redistribution to servers or lists, or to reuse any copyrighted component of this work in other works must be obtained from the IEEE. 


\title{
Resonant-Wavelength Control of Nanocavities by Nanometer-Scaled Adjustment of Two-Dimensional Photonic Crystal Slab Structures
}

\author{
Bong-Shik Song, Takuji Nagashima, Takashi Asano, and Susumu Noda, Member, IEEE
}

\begin{abstract}
In this letter, we experimentally demonstrate resonant-wavelength control of a series of 16 nanocavities in a two-dimensional photonic crystal slab structure by nanometer-order variation of the lattice constants and air-holes sizes. The cavities show a linear dependence on these parameters, a 1-nm increase of lattice constant or air-hole size leading to 4.2-nm increase or 1.56- $\mathrm{nm}$ decrease of the resonant wavelength, respectively. These experimental results are in good agreement with the finite-difference time-domain calculations and have a small standard deviation of wavelength $(\sim 1 \mathrm{~nm})$ between samples on a single chip. These results will stimulate development in areas such as ultrasmall and multichannel filters, sensors, and switches.
\end{abstract}

Index Terms-Nanocavity, optical filters, photonic crystal (PC), sensor.

\section{INTRODUCTION}

$\mathbf{P}$ HOTONIC crystals (PCs) with subwavelength-scaled periodic structure are attracting much interest for their ability to produce nanophotonic devices. Recently, the quality $(Q)$-factors of nanocavities in two-dimensional (2-D) PC slab structures have been increased remarkably [1]-[3]. In general, control over the resonant wavelength of such a high- $Q$ nanocavity is very important for various applications. However, controlling the resonant wavelengths of the PC nanocavities has been a major challenge because of their sensitivity to changes of the PC's geometric parameters such as the lattice constant [4], filling factor [5], and slab thickness [6]. We have demonstrated nanocavities with the resonant wavelength spacing of $5 \sim 6 \mathrm{~nm}$ [4]. However, a quantitative discussion of the wavelength spacing has not yet been given and to date increased accuracy of control over a larger number of channels has not been demonstrated. In this letter, we show experimentally resonant-wavelength control of 16 nanocavities in series by nanometer-scaled adjustment of the lattice constants and air-hole sizes of their PC slab structures. It is also shown that the

Manuscript received September 29, 2007; revised December 29, 2007. This work was supported by a Grant-in-Aid for Scientific Research of Priority Areas from the Ministry of Education, Culture, Sport, Science, and Technology of Japan, and by the Faculty Research Fund, Sungkyunkwan University, 2006.

B. S. Song was with the Department of Electronic Science and Engineering, Kyoto University, Kyoto 615-8510, Japan. He is now with the School of Information and Communication Engineering and Sungkyunkwan Advanced Institute of Nanotechnology, Sungkyunkwan University, Suwon, Gyeonggi-do 440746, Korea (e-mail: songwiz@skku.edu).

T. Nagashima, T. Asano, and S. Noda are with the Department of Electronic Science and Engineering, Kyoto University, Kyoto 615-8510, Japan (e-mail: snoda@kuee.kyoto-u.ac.jp).

Digital Object Identifier 10.1109/LPT.2008.918890

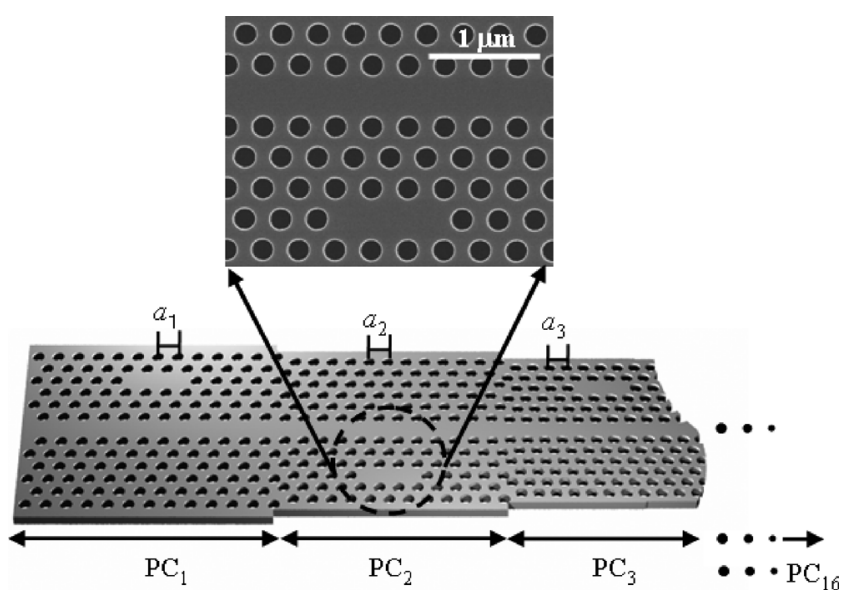

Fig. 1. Schematic of a sample with arrayed $16 \mathrm{PC}$ units with different lattice constants $\left(a_{1}, a_{2}, \ldots, a_{16}\right)$. Individual PC units have a line-defect waveguide and a nanocavity. Inset shows the $\mathrm{SEM}$ image of $\mathrm{PC}_{2}$ unit's top surface near the nanocavity.

experimental results are in good agreement with calculations and have a very small wavelength deviation between several samples on a single chip.

\section{STRUCTURE OF FABRICATED SAMPLES}

Fig. 1 shows the schematic of the fabricated sample. It consists of $16 \mathrm{PC}$ units $\left(\mathrm{PC}_{1}, \mathrm{PC}_{2}, \ldots, \mathrm{PC}_{16}\right)$ with their individual lattice constants $(a)$ determined by $a_{n}=420-1.25 \times(n-1) \mathrm{nm}$ $(n=1,2, \ldots, 16)$. Each PC unit consists of a waveguide made by one line of missing holes and a nanocavity of three missing holes separated from the waveguide by four rows as shown in the inset of Fig. 1. The air-hole size is constant for all the PC units in a sample. The dimensions of each PC unit are as small as $12 \mu \mathrm{m} \times 10 \mu \mathrm{m}$. In each PC unit, the total $Q$-factor of the cavity has been designed to be $\sim 3800$ (i.e., the resonant spectrum has a full-width at half-maximum $\sim 0.4 \mathrm{~nm}$ ) [4]. The sample was fabricated from silicon-on-insulator (SOI) with a 250-nm-thick silicon layer on $3 \mu \mathrm{m}$ of $\mathrm{SiO}_{2}$. The PC patterns were defined in resist using electron-beam lithography (EBL) on SOI. The air-hole diameter is controlled by the dose-time in the EBL system. The patterns were then transferred into the silicon layer by inductively coupled plasma etching. Finally, the $\mathrm{SiO}_{2}$ layer was removed by a HF solution to form an air-bridge structure. Two samples with different air-hole diameters $(d)$ were fabricated: type-A $(d=233.6 \mathrm{~nm})$ and type-B $(d=240.0 \mathrm{~nm})$. The air-hole diameter in each sample was evaluated by statistical analysis of scanning electron microscope [(SEM) Hitachi, 


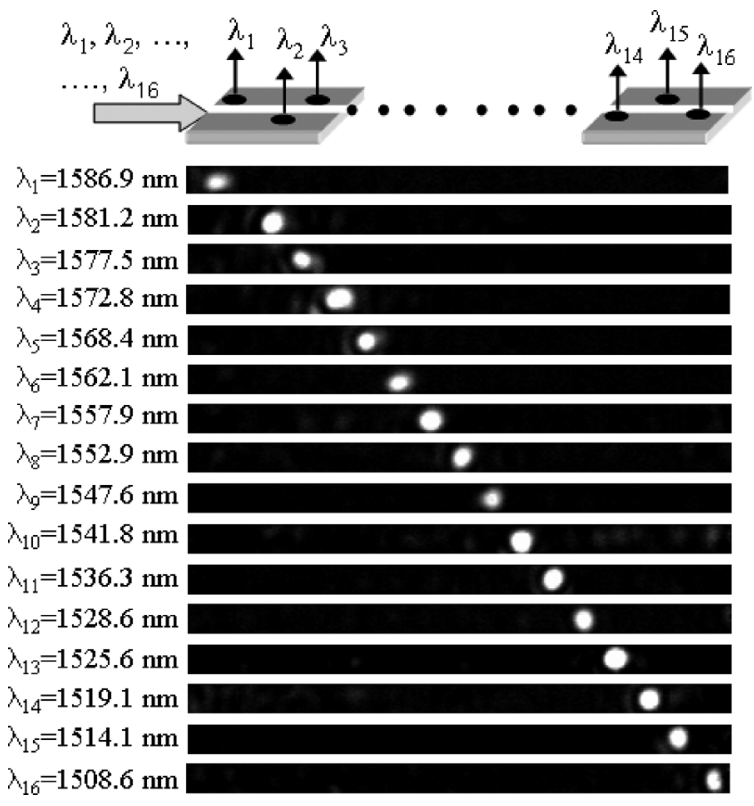

Fig. 2. Emission drop pattern of light from individual cavities in one type-A sample observed by infrared camera: As the wavelength of light entering waveguide is changed from $\lambda_{1}(=1586.9 \mathrm{~nm})$ to $\lambda_{16}(=1506.8 \mathrm{~nm})$, light is dropped by the cavity with the approximate resonant wavelength.

S-4800] images of the fabricated samples using image analysis software (Mediacybernetics, Image-Pro). Three sets of type-A and type-B samples were fabricated on the same chip to make the quantitative discussion of the optical properties possible.

\section{RESULTS AND DISCUSSION}

The optical characteristics of the samples were measured and the relationship between the resonant wavelength and the sample geometry was studied. In order to investigate the optical characteristics, light is coupled into the cleaved facet of the waveguide as schematically shown in the Fig. 2 (top). In a given PC unit, light propagating along the waveguide is coupled to a cavity when its wavelength matches the resonant wavelength of the cavity. The light coupled to the cavity is then emitted into the free space because the cavity mode is leaky. This dropped light emitted from the cavity is observed (or measured) using an infrared camera (or InGaAs photodiode). Fig. 2 shows near-field images of the observed dropped light for a type-A sample where $\lambda_{1}, \lambda_{2}, \ldots, \lambda_{16}$ are the resonant wavelengths of the individual cavities in $16 \mathrm{PC}$ units $\left(\mathrm{PC}_{1}, \mathrm{PC}_{2}, \ldots, \mathrm{PC}_{16}\right)$, respectively. As seen in Fig. 2, light with iteratively shorter wavelengths is emitted in succession from the cavities in PC units, and the spacing of the resonant wavelength between neighboring PCs is $\sim 5 \mathrm{~nm}$. These results imply that the optical properties are controllable by using a lattice-constant difference between the PC regions. The detailed drop spectra for the individual cavities were measured and shown in Fig. 3(a), confirming the wavelength spacing between the neighboring resonant wavelengths is $\sim 5 \mathrm{~nm}$ for all the PC units. This consistent wavelength spacing is almost equal to the wavelength-shifted value expected from a lattice-constant difference of $1.25 \mathrm{~nm}$ according to finite-difference time-domain (FDTD) simulations [7] and implies that 2-D PC slab structures with
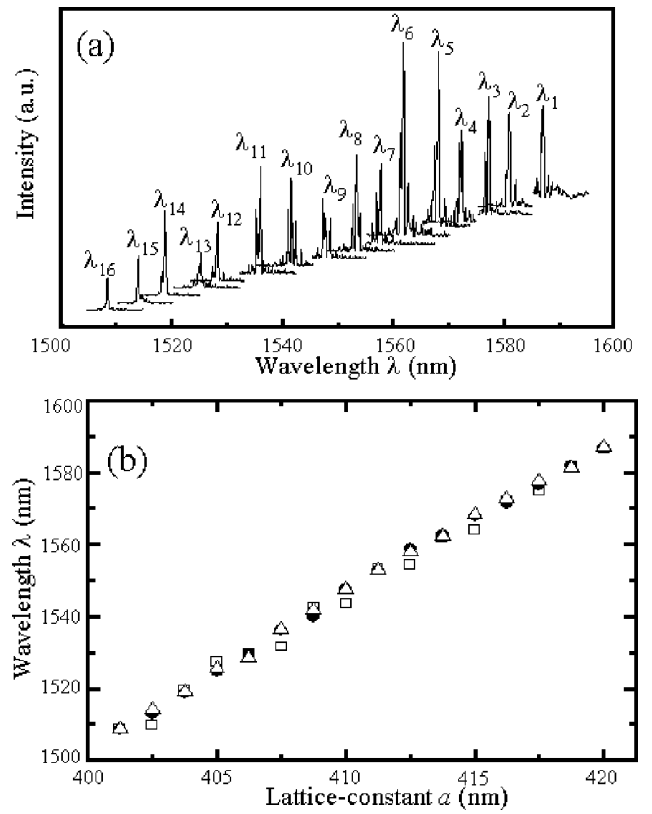

Fig. 3. (a) Detailed drop spectra for the individual cavities in Fig. 2. (b) Plot of resonant wavelengths versus the lattice constant for the three type-A samples.

16 nanocavities can be sufficiently controlled on a nanometer scale. There is nonuniform emission intensity from the cavities and the splitting spectra at resonant wavelengths (especially, longer than $1530 \mathrm{~nm}$ ), which are due to the Fabry-Pérot interferences between the input facet and hetero-interfaces (which are boundaries between PCs with different lattice constants) [8]. The detailed analysis of the interference in this sample will be reported elsewhere. The problem of the Fabry-Pérot interference could be solved by connecting a wire waveguide to the PC waveguides and thus reducing the reflection from input facet [9], [10]. The relationship between the designed lattice constants and the measured resonant wavelengths is plotted in Fig. 3(b) for all three type-A samples fabricated on the same chip. This shows that the resonant wavelengths of all the samples are directly proportional to the lattice constant. The mean of the three samples can be fitted linearly and the slope of the fitted line shows a 4.2-nm increase of wavelength per 1-nm increase of lattice constant, which is in good agreement with the calculations [7]. Furthermore, the standard deviation of the resonant wavelengths for the three samples is within $1.1 \mathrm{~nm}$. This implies that the resonant wavelength is well controlled by nanometer-scale adjustment of the lattice constants in each of the 16 PC units. The deviation of resonant wavelengths is probably due to very slight fluctuation in the lattice constant, air-hole size, and slab thickness.

Because the lattice constant steps of $1.25 \mathrm{~nm}$ used in the above experiment are close to resolution limit in our EBL system, we require another method for finer control over the resonant wavelength. Here, we investigate the influence of changing the air-hole diameter (i.e.,: the filling factor). Fig. 4(a) shows the calculated resonant wavelength of the cavity with three missing holes versus the air-hole diameter, which is calculated by using the 3-D FDTD method assuming lattice constant $a=416.6 \mathrm{~nm}(0.6 a=250 \mathrm{~nm})$. The resonant wavelength of 

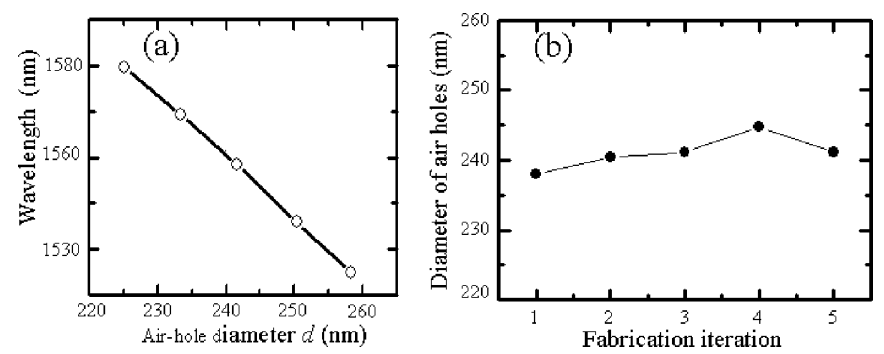

Fig. 4. (a) Calculated result of resonant wavelength of a cavity versus air-hole diameter (b) Air-hole size variation for the successively fabricated samples.

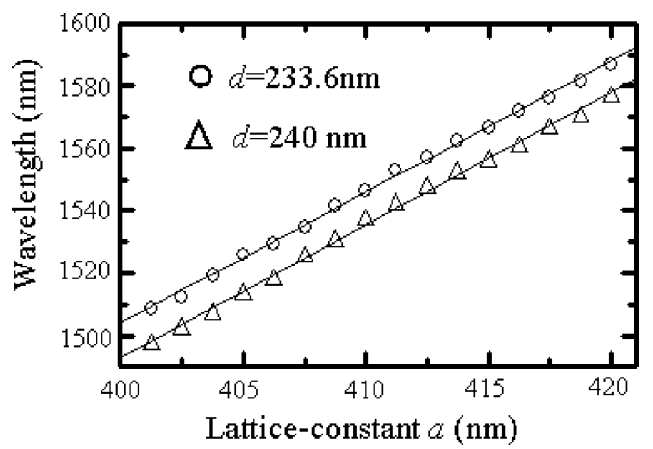

Fig. 5. Measured result of the mean resonant wavelength in the three samples $\mathrm{A}(d=233.6 \mathrm{~nm})$ and $\mathrm{B}(d=240 \mathrm{~nm})$. The two lines represent the fittings of the samples $\mathrm{A}$ and $\mathrm{B}$, respectively.

the cavity changes almost linearly with the air-hole diameter. The slope of the fitted line is -1.34 wavelength (nanometers) per air-holes diameter (nanometers), smaller than the shifting ratio of resonant wavelength by changing the lattice constant. To verify that the diameter can be controlled on a nanometer scale, we examined the air-hole sizes of the five samples with identical design but fabricated separately. Here, the target diameter of the air holes is $240 \mathrm{~nm}$. The air-hole sizes of the five samples are plotted in Fig. 4(b). The standard deviation of air-hole sizes is as small as $2.4 \mathrm{~nm}$, meaning that the air-hole diameter is controllable on a nanometer scale. In particular, the air-hole-diameter difference between iterations 2 and 3 is only $0.8 \mathrm{~nm}$ corresponding to repeatability of $99.6 \%$. On the basis of the above experiment, three type-B samples with an air-hole diameter of $240 \mathrm{~nm}$, which is $6.4 \mathrm{~nm}$ larger than that of the type-A samples, were fabricated on an SOI chip. The resonant wavelengths of cavities in the type-B samples were measured and the mean resonant wavelengths of the cavities are plotted as circles of Fig. 5. The standard deviation of the wavelengths of the three type-B samples was $0.6 \mathrm{~nm}$. As seen in the figure, the resonant wavelengths of the type-B samples are uniformly shifted toward shorter wavelengths by almost $10 \mathrm{~nm}$ while the slope of wavelength versus lattice constant remains. From the figure, the ratio of the wavelength to air-hole diameter is estimated to be 1.56 (= $10 \mathrm{~nm}$ in wavelength $/ 6.4 \mathrm{~nm}$ in air-hole diameter). This is less than half of the ratio of 4.2 (wavelength versus lattice constant). This ratio of 1.56 is almost consistent with the theoretical result of 1.34 shown in Fig. 4(a). Optimization of the lattice constant and air-hole size in 2-D PC structures by the advanced EBL technique of weighted dose allocation for fine-control of pitch used in 1-D gratings [11] and postprocessing for matching a desired resonant wavelength [12] should lead to the realization of ultrasmall 2-D PC samples with multiple wavelength operation and resonant-wavelength spacing of less than $1 \mathrm{~nm}$.

\section{CONCLUSION}

We have demonstrated resonant-wavelength control of 16 arrayed nanocavities by nanometer-scaled differences in lattice constant and air-hole size in a 2-D PC slab. We have shown experimentally that the controlled wavelength ratios are 4.2 and $1.56 \mathrm{~nm}$ per nanometer of change in lattice constant and air-hole diameter, respectively. In addition, we show that the experimental results are in agreement with the FDTD calculations and have a small standard deviation of wavelength $\sim 1 \mathrm{~nm}$ of several samples on a single chip. We believe that these results will stimulate various applications and the developments of ultrasmall optical filter and bio/chemical sensors with a dense wavelength spacing and precise resonant wavelength.

\section{REFERENCES}

[1] Y. Akahane, T. Asano, B. S. Song, and S. Noda, "High-Q photonic nanocavity in a two-dimensional photonic crystal," Nature, vol. 425, no. 6961, pp. 944-947, Oct. 30, 2003.

[2] B. S. Song, S. Noda, T. Asano, and Y. Akahane, "Ultra-high-Q photonic double-heterostructure nanocavity," Nat. Mater., vol. 4, no. 3, pp. 207-210, Mar. 2005.

[3] S. Noda, M. Fujita, and T. Asano, "Spontaneous-emission control by photonic crystals and nanocavities," Nature Photon., vol. 1, pp. 449-458, 2007.

[4] B. S. Song, S. Noda, and T. Asano, "Photonic devices based on in-plane hetero photonic crystals," Science, vol. 400, p. 153, 2003.

[5] G. Subramanina, S. Y. Lin, J. R. Wendt, and J. M. Rivera, "Tuning the microcavity resonant wavelength in a two-dimensional photonic crystal by modifying the cavity geometry," Appl. Phys. Lett., vol. 83, pp. 4491-4493, 2003.

[6] A. R. Alija, L. J. Martinez, A. Garcia-Martin, M. L. Dotor, D. Golmayo, and P. A. Postigo, "Tuning of spontaneous emission of two-dimensional photonic crystal microcavities by accurate control of slab thickness," Appl. Phys. Lett., vol. 86, pp. 141101(1)-(3), 2005.

[7] B. S. Song, T. Asano, Y. Akahaen, Y. Tanaka, and S. Noda, "Multichannel add/drop filter based on in-plane hetero photonic crystals," $J$. Lightw. Technol., vol. 23, no. 3, pp. 1449-1455, Mar. 2005.

[8] B. S. Song, T. Asano, Y. Akahane, and S. Noda, "Role of interfaces in heterophotonic crystals for manipulation of photons," Phys. Rev. B, vol. 71, pp. 195101(1)-(5), 2005.

[9] E. Miyai and S. Noda, "Structural dependence of coupling between a two-dimensional photonic crystals waveguide and a wire waveguide," J. Opt. Soc. Amer. B, Opt. Phys., vol. 21, pp. 67-72, 2004.

[10] Y. A. Vlasv, M. OBoyle, H. F. Hamann, S. J. McNab, and N. Moll, “Active control of slow light on a chip with photonic crystal waveguide," Nature, vol. 438, pp. 66-69, 2005.

[11] Y. Muroya, T. Nakamura, H. Yamada, and T. Torikai, "Precise wavelength control for DFB laser diodes by novel corrugation delineation method," IEEE Photon. Technol. Lett., vol. 9, no. 3, pp. 288-290, Mar. 1997.

[12] K. Hennessy, A. Badolato, A. Tamboli, P. M. Petroff, E. Hua, M. Atature, J. Dreiser, and A. Imamoglu, "Tuning photonic crystal nanocavity modes by wet chemical digital etching," Appl. Phys. Lett., vol. 87, pp. 021108(1)-(3), 2005. 